\title{
PAPER
}

\section{Attenuated nuclear shrinkage in neurones with nuclear inclusions of SCA 1 brains}

\author{
U Nagaoka, T Uchihara, K Iwabuchi, H Konno, M Tobita, N Funata, S Yagishita, T Kato
}

J Neurol Neurosurg Psychiatry 2003;74:597-601

See end of article for authors' affiliations .........................

Correspondence to: Dr T Uchihara, Department of Neuropathology, Tokyo Metropolitan Institute for Neuroscience, 2-6 Musashidai, Fuchu, Tokyo 183-8526, Japan; uchihara@tmin.ac.jp

Received 20 June 2002 In revised form

12 November 2002

Accepted

21 November 2002
Background: Spinocerebellar ataxia type 1 (SCA 1 ) is one of the autosomal dominant neurodegenerative disorders commonly linked to pathological expansion of the CAG repeat of the relevant gene. Nuclear inclusions and neurodegeneration are both triggered by this pathological expansion of the $\mathrm{CAG/polyglutamine} \mathrm{repeat} \mathrm{on} \mathrm{ataxin-1,} \mathrm{but} \mathrm{it} \mathrm{remains} \mathrm{to} \mathrm{be} \mathrm{determined} \mathrm{whether} \mathrm{or} \mathrm{not} \mathrm{nuclear} \mathrm{inclu-}$ sion formation is associated with accelerated neurodegeneration.

Objective: To examine the influence of nuclear inclusions on nuclear size and deformity in human brains from patients suffering from SCA 1 .

Material: Pontine sections of brains obtained at necropsy from seven patients with SCA1 and five controls.

Methods: The size and deformity of each neuronal nucleus was quantified. Nuclei with and without inclusions were examined separately to assess the possible influence of nuclear inclusions on neurodegeneration.

Results: Nuclear shrinkage and deformity were more marked in SCA1 brains than in controls. This shrinkage was attenuated in neurones containing nuclear inclusions.

Conclusions: The existence of nuclear inclusions in SCA 1 is presumably linked to a mechanism that attenuates rather than accelerates nuclear shrinkage. This in vivo finding may provide a clue to constructing a rational therapeutic strategy for combating neurodegeneration associated with nuclear inclusions.
S pinocerebellar ataxia type 1 (SCAl) is an autosomal dominant neurodegenerative disorder characterised by degeneration of the cerebellar Purkinje cells, inferior olive neurones, and neurones within cranial nerve nuclei, leading to progressive ataxia, dysarthria, amyotrophy, and bulbar dysfunction. Symptoms of SCAl typically become apparent in mid-life and worsen over the subsequent 10 to 15 years. The mutations of the gene linked to SCAl and several other autosomal dominant neurodegenerative disorders have been shown to be an expansion of the CAG trinucleotide repeat which results in an abnormally elongated polyglutamine tract in the mutated proteins. The normal alleles of the SCAl gene contain up to 36 CAG repeats, whereas disease alleles have over 43 repeats.

Numerous studies have suggested that the expanded polyglutamine tract leads to neurodegeneration by conferring a toxic gain of function rather than by loss of normal physiological function. One of the hallmarks of these diseases is the formation of insoluble protein aggregates or inclusions, which are found in the brains of affected patients. The inclusions are immunoreactive for ubiquitin and contain expanded polyglutamine, molecular chaperones, and components of the proteasome. ${ }^{23}$ Therefore, it is assumed that these CAG/ polyglutamine repeat disorders share a mechanism underlying nuclear inclusion formation and neurodegeneration, both being triggered by an expansion of CAG/polyglutamine. How the expanded polyglutamine leads to long term neurodegeneration, however, remains unknown, and one of the essential questions is how nuclear inclusion formation is linked to neurodegeneration. ${ }^{4}$

It is still under debate whether nuclear inclusions are associated with accelerated neurodegeneration, or whether they may have a protective function. If nuclear inclusion formation leads to neurodegeneration, it is expected that each individual neurone would show shrinkage once it harbours nuclear inclusions. It is therefore essential to identify possible morphological changes in neurones and their relation to nuclear inclusions in human brains obtained at necropsy. This is the first morphometric study on SCAl designed to examine the influence of nuclear inclusions on nuclear size and deformity in human brains.

\section{METHODS}

We examined brains obtained at necropsy from seven Japanese cases of SCAl and five controls without neurological disorders. Three of the seven SCAl cases were from the same family. Clinical and pathological features of the SCAl cases were compatible with the disease, and the diagnosis was also confirmed by genetic analysis.

Formalin fixed, paraffin embedded sections ( $6 \mu \mathrm{m}$ thick) were obtained from the mid-pons. Pontine nuclei were chosen for this analysis because of the homogeneous size and character of the neurones in the pons, which makes it easier to quantify and interpret morphological alterations. The high frequency of nuclear inclusions in pontine neurones is another advantage for estimating their possible influence. The sections from the pons contained no localised foci of ischaemia or gliosis.

Deparaffinised sections from the SCAl cases were immunostained with an antiubiquitin antibody (rabbit polyclonal, 1:1000; Dako, Glostrup, Denmark), with diaminobenzidine as a chromogen, and were then stained lightly with haematoxylin. Ten rectangular microscopic fields $\left(400 \times 250 \mu \mathrm{m}^{2}=0.1\right.$ $\mathrm{mm}^{2}$ ) were chosen at random and captured by a digital camera connected to a microscope. Large cells harbouring Nissl

Abbreviations: $\mathrm{Cl}$, circularity index; DRPLA, dentatorubral-pallidoluysian atrophy; NIHID, neuronal intranuclear hyaline inclusion disease; $\mathrm{NI}$, nuclear inclusions present; $\mathrm{NI}-$, nuclear inclusions absent; SCA 1, spinocerebellar ataxia type 1 

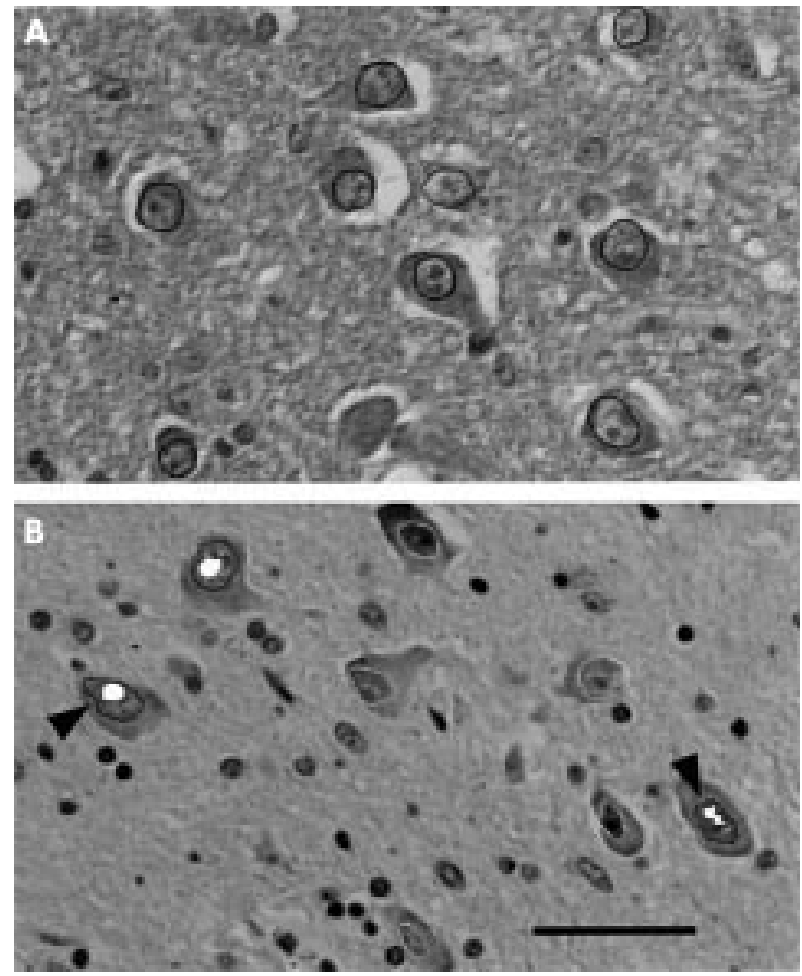

Figure 1 Pontine neurones from a control case (panel A, haematoxylin-eosin stain) and a case of spinocerebellar ataxia type 1 (SCA1) (panel B, ubiquitin immunostain counterstained with haematoxylin). The contour of the nucleus of each neurone is traced in black (in panel $A$, and neurones with nuclear aggregates in panel B) or in white (neurones without nuclear inclusions in panel B). Undulations of the nuclear membrane are conspicuous (arrowheads in panel B) in SCA1 neurones. Ubiquitin immunopositive nuclear inclusions are indicated as white spots in panel $\mathrm{B}$.

substances and nuclei containing identifiable nucleoli or ubiquitin immunopositive nuclear inclusions were identified as neurones. Every field contained neurones with and without nuclear inclusions.

The contours of the nuclear membrane in each neurone and of the nuclear inclusions, if present, were traced on a digitiser coupled to a liquid crystal display (PL-400, Wacom, Saitama, Japan). Examples are shown in fig 1. "Nuclear area," "perimeter," "long axis," and "short axis" from the traced nuclear contours, and "nuclear inclusion area" from the traced nuclear inclusion contours, were calculated using NIH-Image (version 1.62). Because an increase in nuclear size is attributable to the presence of nuclear inclusions, nuclear area not occupied by nuclear inclusions ([nuclear area - nuclear inclusion area]) was also analysed. Nuclear deformity was assessed by the long axis/short axis ratio and "circularity index" (CI), defined by the ratio of two diameters, one of which is estimated from the perimeter (perimeter/2 $\pi$ ) and the other from the area $(\sqrt{ }[\operatorname{area} / \pi) . \mathrm{CI}=1$ if the traced nuclear contour is an exact circle. An increase in the long axis/short axis ratio or CI is correlated with the severity of nuclear deformities (elongation, distortion, or undulations of the nuclear membrane).

\section{Statistics}

Differences in the variables between groups with nuclear inclusions (NI+) and groups without nuclear inclusions (NI-) from both SCAl cases (SCAl total group, including both $\mathrm{NI}+$ and NI- groups) and controls were estimated by analysis of variance (ANOVA) or Student's $t$ test.

\section{RESULTS}

Table 1 shows the demographic data on the patients and the neuronal count of each case. The mean of the neuronal counts (representing the mean packing density) of the SCAl group $\left(83.9 / \mathrm{mm}^{2}\right)$ was significantly smaller than that of the controls (160.2/ $\mathrm{mm}^{2}, \mathrm{p}<0.01$; Mann-Whitney U test).

The morphological data assembled from each group are shown in table 2. Nuclear area, perimeter, and short axis were significantly smaller in the SCAl total group than in the controls (for example, the mean nuclear area was $81.4 \mu \mathrm{m}^{2}$ in the SCAl group $v 90.5 \mu \mathrm{m}^{2}$ in the control group; $\mathrm{p}<0.0001$ by Student's $t$ test).

The inverted histograms in fig 2 show that the size distribution (nuclear area) of the SCAl total group (filled bars) was smaller than that of the controls (unfilled bars). Nuclear shrinkage (estimated by nuclear area, perimeter, long axis, and short axis) was more profound in the NI- group than in the $\mathrm{NI}+$ group, or the neuronal nuclei in the NI+ group were less atrophic than in the NI- group $(p<0.0001$ by ANOVA, PLSD (protected least significant difference) of Fisher at 1\% probability). The upper histograms in fig 2 show the difference in nuclear area between the NI+ group (unfilled bars) and the NI- group (filled bars).

There was no difference between the NI+ group and the NI- group in [nuclear area - nuclear inclusion area], neither was there a significant correlation between nuclear inclusion area and nuclear area, as shown in the scattergram in fig 3

Table 1 Neuronal counts $\left(/ \mathrm{mm}^{2}\right)$ with and without nuclear inclusions

\begin{tabular}{llllll}
\hline Case & $\begin{array}{l}\text { Age at onset/death } \\
\text { (years) }\end{array}$ & Sex & $\begin{array}{l}\text { Inclusions present } \\
\left(\mathbf{n} / \mathrm{mm}^{2}\right)\end{array}$ & $\begin{array}{l}\text { Inclusions absent } \\
\left(\mathbf{n} / \mathrm{mm}^{2}\right)\end{array}$ & Total \\
\hline SCA1 case 1 & $39 / 55$ & $\mathrm{~F}$ & 24 & 71 & 95 \\
SCA1 case 2 & $41 / 50$ & $\mathrm{M}$ & 29 & 67 & 96 \\
SCA1 case 3 & $41 / 55$ & $\mathrm{~F}$ & 32 & 62 & 94 \\
SCA1 case 4 & $30 / 50$ & $\mathrm{~F}$ & 23 & 67 & 90 \\
SCA1 case 5 & $46 / 63$ & $\mathrm{~F}$ & 14 & 40 & 54 \\
SCA1 case 6 & $37 / 48$ & $\mathrm{M}$ & 23 & 49 & 72 \\
SCA1 case 7 & $46 / 66$ & $\mathrm{M}$ & 12 & 74 & 86 \\
Mean & & & & & $83.9^{*}$ \\
& & & & 140 & 140 \\
Control & $/ 25$ & $\mathrm{M}$ & 0 & 174 & 174 \\
Control & $/ 25$ & $\mathrm{~F}$ & 0 & 154 & 154 \\
Control & $/ 40$ & $\mathrm{~F}$ & 0 & 157 & 157 \\
Control & $/ 44$ & $\mathrm{M}$ & 0 & 176 & 176 \\
Control & $/ 45$ & $\mathrm{~F}$ & 0 & & 160.2 \\
Mean & & & & & \\
\hline
\end{tabular}

*Significantly smaller than the control group ( $p<0.01$; Mann-Whitney $U$ test).

$F$, female; $M$, male; SCA I, spinocerebeller ataxia type 1 . 
Table 2 Morphometric indices of pontine neurones in control and SCA1 cases

\begin{tabular}{|c|c|c|c|c|}
\hline \multirow[b]{2}{*}{ Index } & \multirow[b]{2}{*}{ Controls $(n=5)$} & \multicolumn{3}{|l|}{ SCA1 $(n=7)$} \\
\hline & & Total & $\mathrm{NI}+$ & $\mathrm{NI}-$ \\
\hline Number of neurones & 801 & 587 & 157 & 430 \\
\hline Nuclear area $\left(\mu \mathrm{m}^{2}\right)$ & 90.5 (19.8) & $81.4(21.8)^{*}$ & $87.2(24.4)$ & $79.2(20.4)^{* * *}$ \\
\hline $\begin{array}{l}\text { [Nuclear area minus nuclear } \\
\text { inclusion area] }\end{array}$ & & $78.0(21.4)^{*}$ & $74.5(23.6)^{* * *}$ & $79.2(20.4)^{* * *}$ \\
\hline Perimeter $(\mu \mathrm{m})$ & $36.3(4.0)$ & $34.4(4.5)^{*}$ & $35.8(4.9)$ & $34.0(4.3)^{* * *}$ \\
\hline Long axis ( $\mu \mathrm{m})$ & $12.00(1.48)$ & $11.77(1.72)$ & $12.09(1.71)$ & $11.65(1.71)^{* *} \dagger$ \\
\hline Short axis ( $\mu \mathrm{m})$ & $9.54(1.25)$ & $8.71(1.48)^{*}$ & $9.06(1.69)^{* * *}$ & $8.58(1.37)^{* * *} \mathbb{9}$ \\
\hline Long axis/short axis ratio & $1.27(0.18)$ & $1.38(0.25)^{*}$ & $1.37(0.25)^{* * *}$ & $1.38(0.25)^{* * *}$ \\
\hline Circularity index & $1.085(0.037)$ & $1.088(0.040)$ & $1.095(0.048)^{* *}$ & $1.086(0.037)$ \\
\hline
\end{tabular}

Values are expressed as mean (SD)

${ }^{*} \mathrm{p}<0.0001 v$ control group (Student's $t$ test).

${ }^{* *} p<0.01,{ }^{* * *} p<0.0001 v$ control group; $9 p \leqslant 0.0001 v N I+$ group; $\uparrow p<0.01 v N I+$ group (ANOVA, PLSD of Fisher at $1 \%$ probability)

ANOVA, analysis of variance; $\mathrm{NI}$, neurones with nuclear aggregates from SCAl cases; $\mathrm{NI}$-, neurones without nuclear aggregates from $\mathrm{SCA} 1$ cases,

PLSD, protected least significant difference; SCA1, spinocerebellar ataxia type 1; total, all neurones from SCA1 cases.

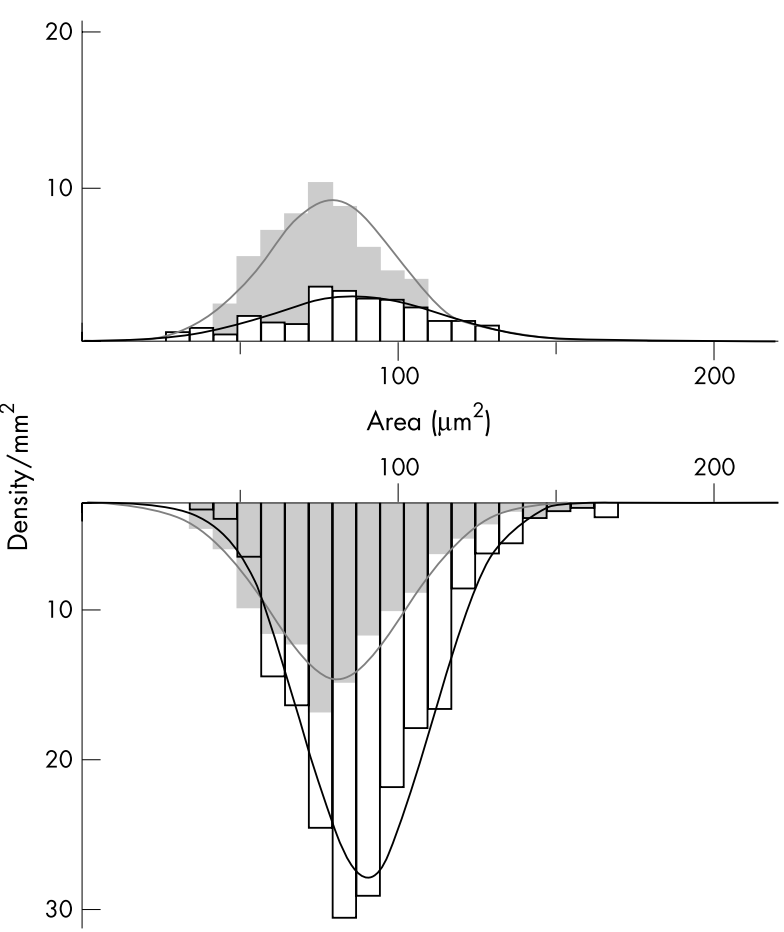

Figure 2 Size distribution of the nuclear size of the pontine neurones from cases with spinocerebellar ataxia type 1 (SCA1) and control cases. Nuclear size estimated as nuclear area in ${\mu m^{2}}^{2}$ is expressed along the abscissa. The ordinate represents relative frequency of each size expressed as density $/ \mathrm{mm}^{2}$. The lower inverted histograms refer to the controls (unfilled bars and a black line) and to the SCA 1 total group (filled bars and a grey line). The upper histograms refer to the SCA 1 group without nuclear inclusions (filled bars and a grey line), and to the SCA1 group with nuclear inclusions (unfilled bars and a black line).

$\left(R^{2}=0.064\right)$. Furthermore, there were no significant correlations between age at onset or duration of illness and any of the morphological variables, including nuclear area (data not shown).

Although the value for long axis was not statistically different between the SCAl total group and the controls, it was significantly smaller in the NI- group than in the NI+ group. The long axis/short axis ratio-one of the indices of nuclear deformity-was increased in both the SCAl groups compared with the controls, but with no significant difference between the NI+ and the NI- groups. CI, another index of nuclear deformity, was significantly increased in the NI+ group com-

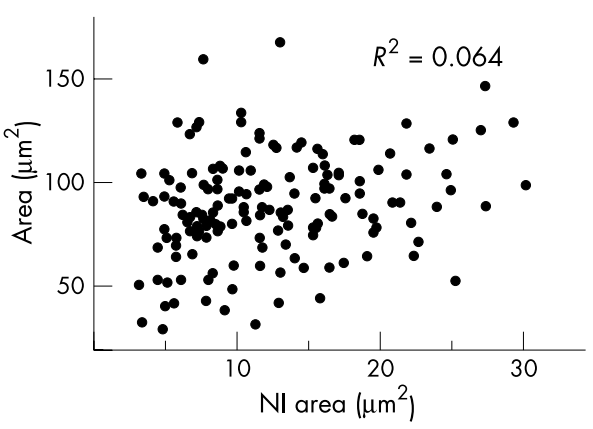

Figure 3 Absence of correlation $\left(R^{2}=0.064\right)$ between nuclear size ("area" in $\mu^{2}$ along the ordinate) and nuclear inclusion size ("NI area" in $\mu \mathrm{m}^{2}$ along the abscissa).

pared with the controls, but the CI value in the NI- group was similar to that in the control group (fig 4).

\section{DISCUSSION}

In this study we investigated whether the presence of nuclear inclusions was linked to morphological changes in neurones during neurodegeneration in human brains obtained at necropsy. Unexpectedly, we found that nuclear shrinkage of the pontine neurones in brains from SCAl patients was attenuated when nuclear inclusions were present. Nuclear inclusions are a pathological hallmark for most of the CAG/polyglutamine repeat disorders, including SCAl, and are composed of the protein altered by the expansion of $\mathrm{CAG} /$ polyglutamine tracts. These altered proteins have therefore been considered to be a link between neurodegeneration and nuclear inclusion formation, although the precise relation between nuclear inclusions and neurodegeneration is still unclear. On the other hand, cellular atrophy-which is considered to represent a stage preceding cell death-is a principal feature of neurodegeneration regardless of its aetiology, because degenerative processes in general develop slowly and usually take several years or even decades.

The nuclear shrinkage in pontine neurones of the SCAl cases observed in our study indicated that cellular atrophy was the pathological feature representing the neurodegenerative process in this condition. Sorting the neurones into those with and without nuclear inclusions further clarified the situation, in that we could show that neurones with nuclear inclusions were less atrophic than those without. This result, from human brains obtained at necropsy, is not compatible with the hypothesis that the neurodegenerative process is accelerated in the presence of nuclear inclusions.

Studies carried out both in vitro and in vivo have provided conflicting evidence about the possible role of nuclear inclusion 


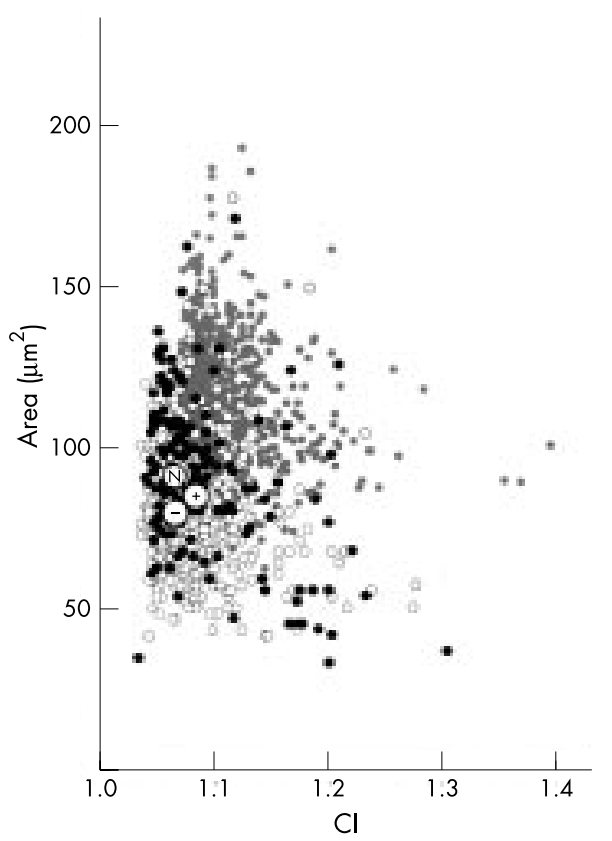

Figure 4 Relation between nuclear size ("area" along the ordinate in $\mu^{2}$ ) and deformity of the nucleus (circularity index $(\mathrm{Cl})$ along the abscissa). $\mathrm{Cl}=1$ if the nucleus is an exact circle and increases if the nucleus is deformed by elongation or undulation. Grey dots $=$ normal group; black dots $=\mathrm{NI}+$ group; white dots $=\mathrm{NI}-$ group. Mean values for each group are plotted within larger circles as: $\mathrm{N}$ (normal group), - (NI- group), and + (NI+ group) in the scattergram. $\mathrm{NI}$, nuclear inclusions.

formation and its relation to neurodegeneration. Neurodegeneration and nuclear inclusion formation in transgenic models of Huntington's disease are induced by the continued expression of the huntingtin fragment, carrying expanded polyglutamine, and are possibly reduced by inhibition of caspase- $1 .{ }^{6}$ However, the suppression of nuclear inclusion formation by a caspase inhibitor did not increase neuronal survival in cultured cells transfected with the huntingtin gene carrying an expanded CAG repeat. ${ }^{7}$ In cultured cells transfected with mutant huntingtin, blocking ubiquitination led to decreased nuclear inclusion formation and accelerated cell death. ${ }^{8}$ This discrepancy is reflected in the observation that transgenic mice expressing mutant ataxin-1 without the self association domain develop a pathological phenotype without forming nuclear inclusions.?

Necropsy observations on human brains from patients with Huntington's diseas $\mathrm{e}^{10}$ or neuronal intranuclear hyaline inclusion disease (NIHID) ${ }^{11}$ showed a similar discrepancy between neuronal depletion and nuclear inclusion formation. At least two entirely opposing interpretations have been proposed to explain these data. If the presence of nuclear inclusions correlates with mutant protein induced cell death, atrophic features may be more exaggerated in neurones containing nuclear inclusions than in those without. The other possibility is that nuclear inclusion formation may be part of a process linked to the protection of cells from the toxic effects of the mutant protein. In that case we may expect that neurones without nuclear inclusions would show more atrophic features. The former possibility is not in agreement with what we observed in pontine neurones of the SCAl brains in the present study, because neuronal nuclei containing nuclear inclusions were not smaller than those without, though pontine neurones of SCAl brains-regardless of the presence of nuclear inclusions-were more atrophic than control brains.

When "nuclear inclusion area" was subtracted from "nuclear area," the size of neuronal nuclei in the NI+ group did not differ from that in the NI- group. However, nuclear inclusion size was not correlated with nuclear area. This means that the influence of nuclear inclusions on nuclear area-even if the inclusions were inserted from the extranuclear compartment-does not solely reflect the simple addition of "nuclear inclusion area." Nuclear inclusions may in fact be formed in the nucleus rather than being inserted from extranuclear compartments. In that case, a fraction of the nucleus is altered into nuclear inclusions, in which case one might expect that [nuclear area-nuclear inclusion area] would be decreased by the size of the nuclear inclusions. Our morphometric study, however, showed that the decrease in [nuclear area - nuclear inclusion area] in the NI+ group relative to the NI- group $\left(79.2 \mu^{2}-74.5 \mu^{2}=4.7 \mu^{2}\right)$ was less than half the nuclear inclusion area itself $\left(87.2 \mu^{2}-74.5 \mu^{2}=12.7\right.$ $\mu^{2}$ ). If nuclear inclusion formation were linked to some mechanism accelerating degeneration, the decrease in [nuclear area-nuclear inclusion area] should have exceeded the nuclear inclusion area. The present study showed, on the contrary, that this decrease was much attenuated. This attenuated decrease is explicable if nuclear size as a whole is increased during nuclear inclusion formation, as we demonstrated in this study.

A recent morphometric study showed that in dentatorubral-pallidoluysian atrophy (DRPLA), cerebellar granule cells containing nuclear inclusions were larger than those that did not. ${ }^{12}$ Our recent morphometric study on pontine neurones from Machado-Joseph disease brains showed that [nuclear area - nuclear inclusion area] of NI+ neurones was significantly larger than that of NI- neurones. ${ }^{13}$ Furthermore, it was even larger in NIHID than in normal controls. ${ }^{14}$ In these disorders, NI+ neurones were in general less atrophic than NI- neurones, raising the possibility that neurones are equipped with common machinery triggered by or accompanied by nuclear inclusion formation and possibly counteracting polyglutamine induced neurodegeneration. However, the influence of nuclear inclusions on nuclear size is variable depending on the disease involved, and they appear to have a relatively less pronounced influence in SCAl. If this were not the case, the atrophic process in SCAl might be so profound as to overwhelm this possible counteracting factor.

While it is generally recognised that CAG repeat length relates to the severity of illness and the age of onset, the age at onset of the SCAl cases in this study was restricted within the range of 30 to 46 years and there was no significant correlation between the age at onset and the different variables that we examined. Larger numbers of cases with a broader age range of onset might have identified a possible influence of age on morphological change in the two groups of neurones. However, it may be that age at onset is not a major determinant for nuclear inclusion formation and neuronal atrophy.

In the present study, a fraction of neurones that actually contained nuclear inclusions might have been falsely classified into the NI- group because our observation on a two dimensional plane may have failed to detect nuclear inclusions that were not included in the plane of the $6 \mu \mathrm{m}$ thick sections. If neurones with nuclear inclusions were smaller than those without nuclear inclusions, this possible failure to identify inclusions not recognised in the two dimensional plane might have led to underestimation of the size of the neurones in the NI- group. However, because the present study indicated the opposite-that is, it showed that neuronal nuclei in the NI+ group were larger than those in the NIgroup-it is reasonable to conclude from this two dimensional study that neuronal nuclei with nuclear inclusions are significantly larger than neuronal nuclei without nuclear inclusions.

Our study showed an increase in the long axis/short axis ratio in the SCAl group and an increase in CI in the NI+ group compared with the controls, without an increase in CI in the NI- group. This suggests that the nuclei of pontine neurones in SCAl are deformed, and especially those with nuclear 
inclusions. One would expect these deformities to be secondary to nuclear shrinkage. The larger nuclear size of the NI+ group, however, was associated with a more marked increase in the indices of nuclear deformity, which indicates that a mechanism other than nuclear shrinkage could be one of the determinants of these deformities. These deformities might otherwise be influenced by the presence of nuclear inclusions in SCAl brains. An apparent indentation of the nuclear membrane may be observed even in normal neurones, ${ }^{15}$ and a pathological increase in this indentation has been described in human brains with DRPLA ${ }^{12}$ and Huntington's disease, ${ }^{16}$ and in an animal model. ${ }^{17}$ Although our present observations, based on light microscopy, failed to identify deep indentations of the nuclear membrane, an increase in the long axis/short axis ratio in SCAl neurones and an increase in the circularity index in SCAl neurones with nuclear inclusions may represent similar pathological changes. These would be common to the CAG/polyglutamine repeat disorders and would be partly independent of nuclear atrophy, as we demonstrated in SCA3 and NIHID brains. ${ }^{12}{ }^{13}$

Although it is evident that both nuclear inclusion formation and neuronal degeneration are induced by an expanded CAG repeat, our study shows that the presence of nuclear inclusions does not necessarily parallel the extent of neurodegeneration, as measured by nuclear size. Rather, the presence of nuclear inclusions was associated with an incease in the size of the neuronal nuclei, which does not support the hypothesis that the inclusions are linked to accelerated neurodegeneration. Cellular mechanisms linking nuclear inclusion formation and neurodegeneration, however, remain elusive. One possibility is that the ubiquitin-proteasome pathway is involved in both neuroprotection and nuclear inclusion formation. Downregulation of ubiquitinproteasome pathway has been found to suppress nuclear inclusion formation and to accelerate cell death in parallel in studies carried out in vitro. ${ }^{18}$ This inverse relation between cell death and nuclear inclusion formation through the ubiquitinproteasome pathway suggests that nuclear inclusion formation is linked to a possible intrinsic neuroprotective mechanism mediated by ubiquitin. We have shown that ubiquitin is commonly localised to the periphery of nuclear inclusions in brains from patients with Machado-Joseph disease ${ }^{19}$ and NIHID. ${ }^{20}$. These findings are explicable if nuclear inclusion formation mediated by ubiquitin is associated with a mechanism counteracting neuronal shrinkage, as was reported in SCAl transgenic mice. ${ }^{21}$ Further investigation into the relation between nuclear inclusion formation and cellular atrophy will clarify whether these morphological features and their interpretation are shared with other conditions characterised by nuclear inclusions, and will determine whether nuclear inclusions serve as a morphological hallmark for polyglutamine induced neurodegeneration or neuroprotection. Furthermore, clarification of the molecular mechanism of nuclear inclusion formation will give a rational basis for a therapeutic strategy that may retard or even reverse neurodegeneration by activating or modulating this machinery.

\section{ACKNOWLEDGEMENTS}

This work was supported partly by grants from the Ministry of Health and Welfare (KI) and from Japan Society for the Promotion of Science/INSERM (TU).

\section{Authors' affiliations}

U Nagaoka, Department of Neurology, Tokyo Metropolitan Neurological Hospital, Tokyo, Japan

U Nagaoka, T Uchihara, Department of Neuropathology, Tokyo

Metropolitan Institute for Neuroscience

N Funata, Department of Pathology, Tokyo Metropolitan Komagome Hospital

K Iwabuchi, Department of Neurology and Psychiatry, Kanagawa

Rehabilitation Centre, Atsugi, Japan

S Yagishita, Department of Pathology, Kanagawa Rehabilitation Centre

H Konno, Department of Neurology, National Sanatorium Nishitaga

Hospital, Miyagi, Japan

M Tobita, Department of Neurology, Miyagi National Hospital

T Kato, Third Department of Internal Medicine, Yamagata University

School of Medicine, Yamagata, Japan

Competing interests: none declared

\section{REFERENCES}

1 Orr HT, Chung MY, Banfi S, et al. Expansion of an unstable trinucleotide CAG repeat in spinocerebellar ataxia type 1. Nat Genet 1993;4:221-6.

2 Zoghbi HY, Orr HT. Glutamine repeats and neurodegeneration. Annu Rev Neurosci 2000;23:217-47.

3 Kaytor M, Warren S. Aberrant protein deposition and neurological disease. J'Biol Chem 1999;274:37507-10.

4 Sisodia SS. Nuclear inclusions in glutamine repeat disorders: are they pernicious, coincidental, or beneficial? Cell 1998;95: 1-4.

5 Yamamoto A, Lucas JJ, Hen R. Reversal of neuropathology and motor dysfunction in a conditional model of Huntington's disease. Cell 2000;101:57-66.

6 Ona VO, Li M, Vonsattel JP, et al. Inhibition of caspase-1 slows disease progression in a mouse model of Huntington's disease. Nature 1999;399:263-7

$7 \mathrm{Kim} \mathrm{M}$, Lee H-S, LaForet G, et al. Mutant huntingtin expression in clonal striatal cells: dissociation of inclusion formation and neuronal survival by caspase inhibition. J Neurosci 1999;19:964-73.

8 Saudou F, Finkbeiner S, Devys D, et al. Huntingtin acts in the nucleus to induce apoptosis but death does not correlate with the formation of intranuclear inclusions. Cell 1998;95:55-66.

9 Klement IA, Skinner PJ, Kaytor MD, et al. Ataxin-1 nuclear localization and aggregation: role in polyglutamine-induced disease in SCA 1 transgenic mice. Cell 1998;95:41-53.

10 Kuemmerle S, Gutekunst C-A, Klein AM, et al. Huntingtin aggregates may not predict neuronal death in Huntington's disease. Ann Neurol 1999:46:842-9.

11 Takahashi J, Tanaka J, Arai K, et al. Recruitment of non-expanded polyglutamine proteins in intranuclear aggregates of neuronal intranuclear hyaline inclusion disease. J Neuropathol Exp Neurol 2001;60:369-76.

12 Takahashi H, Egawa S, Piao Y-S, et al. Neuronal nuclear alterations in dentatorubral-pallidoluysian atrophy: ultrastructural and morphometric studies of the cerebellar granule cells. Brain Res 2001;919:12-19.

13 Uchihara T, Iwabuchi K, Funata N, et al. Attenuated nuclear shrinkage in neurons with nuclear aggregates - a morphometric study on pontine neurons of Machado-Joseph disease brains. Exp Neurol 2002; 178: 124-8.

14 Uchihara T, Tanaka J, Funata N, et al. Influence of intranuclear inclusion on nuclear size - morphometric study on pontine neurons of neuronal intranuclear inclusion disease cases. Acta Neuropathol 2003; 105: 103-8

15 Roos RAC, Bots GTAM. Nuclear membrane indentations in Huntington's chorea. J Neurol Sci 1983:61:37-47.

16 Bots GTAM, Bruyn GW. Neuropathological changes of the nucleus accumbens in Huntington's chorea. Acta Neuropathol 1981:55:21-2.

17 Davies SW, Turmaine M, Cozens BA, et al. Formation of neuronal intranuclear inclusions underlies the neurological dysfunction in mice transgenic for the HD mutation. Cell 1997;90:537-48

18 Cummings CJ, Reinstein E, Sun Y, et al. Mutation of the E6-AP ubiquitin ligase reduces nuclear inclusion frequency while accelerating polyglutamine-induced pathology in SCA1 mice. Neuron 1999;24:879-92.

19 Fujigasaki H, Uchihara T, Koyano S, et al. Ataxin-3 is translocated into the nucleus for the formation of intranuclear inclusions in normal and Machado-Joseph disease brains. Exp Neurol 2000;1 165:248-56.

20 Takahashi J, Fukuda T, Tanaka J, et al. Neuronal intranuclear hyaline inclusion disease with polyglutamine immunoreactive inclusions. Acta Neuropathol 2000:99:589-94.

21 Cummings CJ, Mancini MA, Antalffy B, et al. Chaperone suppression of aggregation and altered subcellular proteasome localization imply protein misfolding in SCA1. Nat Genet 1998;19:148-54. 